\title{
Levantamento bibliográfico acerca dos fatores que influenciam o desmame precoce
}

\section{Bibliographic survey on the factors that influence early weaning}

Revisión de la literatura sobre las causas del destete temprano

Rafael Radison Coimbra Pereira da Silva ORCID: https://orcid.org/0000-0002-8947-088X Universidade Estadual do Piauí, Brasil E-mail: rafaelcoimbra739@gmail.com

Woodyson Welson Barros da Silva Batista ORCID: https://orcid.org/0000-0002-8451-9795 Universidade Federal do Piauí, Brasil E-mail: woodywb2020@outlook.com

Jaynne da Costa Abreu de Sousa ORCID: https://orcid.org/0000-0002-6152-6052 Centro Universitário Santo Agostinho, Brasil E-mail: jaynnemitchel@gmail.com

Erica Williams de Moreira Lima ORCID: https://orcid.org/0000-0003-3975-5699

Centro Universitário Uninovafapi, Brasil

E-mail: williams-erica1992@outlook.com

Alexsandra Santos Sampaio

ORCID: https://orcid.org/0000-0003-1160-884X Universidade Federal do Delta do Parnaíba, Brasil

E-mail: alexsandrasantossam@gmail.com

Angélica Ribeiro do Nascimento Oliveira ORCID: https://orcid.org/0000-0002-7240-2004 Centro Universitário Maurício de Nassau, Brasil

E-mail: angelicalribeiro.ar19@gmail.com

Gedelvani Francisco Oliveira da Silva ORCID: https://orcid.org/0000-0002-3591-1408 Christus Faculdade do Piauí, Brasil E-mail: gedelvanioliveira@gmail.com

José Marcos Fernandes Mascarenhas ORCID: https://orcid.org/0000-0002-2604-4330 Christus Faculdade do Piauí, Brasil

E-mail: zemarcosmascarenhas@gmail.com

Kelly Emanuelle de Sousa Araújo Santos ORCID: https://orcid.org/0000-0002-6936-0966

Universidade Federal do Maranhão, Brasil

E-mail: emanuellearaujo13@yahoo.com.br Gabriela Maria de Sousa Rodrigues ORCID: https://orcid.org/0000-0002-8053-5536 Universidade Estadual do Piauí, Brasil

E-mail: gabrielarodrigues14_@hotmail.com

Ana Clara dos Santos e Silva Costa ORCID: https://orcid.org/0000-0003-0267-3340 Universidade Federal do Piauí, Brasil E-mail: anaclara369@hotmail.com

Cidianna Emanuelly Melo do Nascimento ORCID: https://orcid.org/0000-0001-5477-4413 Universidade Estadual do Ceará, Brasil

E-mail: profa.cidianna.melo@gmail.com

\section{Resumo}

Objetivo: Identificar na literatura os fatores que favorecem o desmame precoce. Metodologia: Este estudo trata-se de uma revisão integrativa da literatura realizada entre o período de Janeiro a Abril de 2021. Utilizaram-se os descritores: Aleitamento materno, Desmame precoce e Pré-Natal. Os critérios de inclusão foram estudos publicados nos últimos oito anos (2013-2021), estudos originais publicados no Brasil, nos idiomas português, inglês e espanhol e apresentar texto completo. Os critérios de exclusão foram artigos repetidos e/ou que não se encaixam na temática abordada. Resultados: Evidenciou-se, entre os principais fatores que levam ao desmame precoce, a gravidez na adolescência, mãe com baixa produção de leite e condições socioeconômicas desfavoráveis. Conclusão: O pré-natal realizado de forma ideal é a melhor estratégia de promoção ao aleitamento materno exclusivo e prevenção do desmame precoce 
servindo para identificar pessoas em situação de vulnerabilidade e também como forma de intervenção através do acompanhamento e da educação em saúde.

Palavras-chave: Aleitamento materno; Desmame precoce; Pré-natal.

\begin{abstract}
Objective: To identify in the literature the factors that favor early weaning. Methodology: This study is an integrative review of the literature carried out between January and April 2021. The descriptors were used: Breast Feeding, Weaning and Prenatal Care. The inclusion criteria were studies published in the last eight years (2013-2021), original studies published in Brazil, in Portuguese, English and Spanish and presentation of the full text. The exclusion criteria were repeated articles and / or that do not fit the theme addressed. Results: Among the main factors that lead to early weaning, teenage pregnancy, a mother with low milk production and unfavorable socioeconomic conditions were evidenced. Conclusion: Prenatal care performed ideally is the best strategy for promoting exclusive breastfeeding and preventing early weaning, serving to identify people in vulnerable situations and also as a form of intervention through monitoring and health education.
\end{abstract}

Keywords: Breast feeding; Weaning; Prenatal care.

\title{
Resumen
}

Objetivo: Identificar en la literatura los factores que favorecen el destete temprano. Metodología: Este estudio es una revisión integradora de la literatura realizada entre enero y abril de 2021. Se utilizaron los descriptores: Lactancia Materna, Destete y Atención Prenatal. Los criterios de inclusión fueron estudios publicados en los últimos ocho años (2013-2021), estudios originales publicados en Brasil, en portugués, inglés y español y presentación del texto completo. Los criterios de exclusión fueron artículos repetidos y / o que no se ajustan a la temática abordada. Resultados: Entre los principales factores que conducen al destete temprano, se evidenció el embarazo adolescente, una madre con baja producción de leche y condiciones socioeconómicas desfavorables. Conclusión: La atención prenatal realizada de manera ideal es la mejor estrategia para promover la lactancia materna exclusiva y prevenir el destete precoz, sirviendo para identificar a las personas en situación de vulnerabilidad y también como una forma de intervención a través del seguimiento y la educación para la salud.

Palabras clave: Lactancia materna; Destete; Atención prenatal.

\section{Introdução}

O Ministério da Saúde (MS) define o aleitamento materno como uma estratégia que além de promover o vínculo e o afeto entre mãe e filho, é uma intervenção que fornece nutrição de baixo custo e que reduz a morbimortalidade infantil. O Aleitamento materno deve ser exclusivo (AME) até os primeiros seis meses de vida e complementado até os 02 anos de idade da criança (Brasil, 2015).

Silva (2013) afirma que o aleitamento materno provoca benefícios únicos, principalmente em recém-nascidos prétermos, pois favorece o aumento dos índices de inteligência, acuidade visual, melhora da defesa do organismo, visto que no leite materno é passado de mãe para filho imunoglobulinas $G$, além de conferir melhor digestão e ausência de fatores alergênicos, entre outras vantagens e proteções ofertadas pelo aleitamento materno.

No Brasil, algumas dificuldades são identificadas como fatores que as mães utilizam para justificar a interrupção da amamentação exclusiva. Estudo realizado no Paraná identificou, entre os principais motivos para o desmame precoce, a volta ao trabalho, pois a licença maternidade só tem validade de 120 dias e é inferior ao período preconizado de aleitamento materno exclusivo - AME. Além disso, também foram citados fatores biológicos como mamilo invertido, pega incorreta, dor e presença de fissuras mamilar (Peres et al., 2021).

Outro motivo que leva ao desmame precoce é a falta de informação que devem ser fornecidas às mães durante a realização das consultas de pré-natal. A pesquisa realizada por Alencar et al. (2017) evidenciou a importância da orientação prestada as gestantes durante o pré-natal e encontrou associação positiva na adesão às orientações recebidas e maior participação do paciente no seu autocuidado e no cuidado com os recém- nascidos.

Considerando o surgimento dos inúmeros fatores que favorecem ao desmame cada vez mais precoce, busca-se desenvolver este estudo a fim de oferecer suporte para elaboração de estratégias que reduzam esse fato, visto que, o aleitamento materno é de extrema importância para o crescimento saudável da criança. 


\section{Metodologia}

Este estudo é uma revisão integrativa da literatura, a qual se configura como um método que possibilita a síntese de vários estudos relevantes, publicados no meio científico, além de permitir a identificação de lacunas do conhecimento sobre determinado tema. No desenvolvimento desta pesquisa foram adotadas as fases que tangem a revisão integrativa da literatura, a saber: elaboração da pergunta norteadora, pesquisa na literatura, coleta de dados, análise crítica dos estudos incluídos, discussão dos resultados e apresentação da revisão integrativa (Sousa et al. 2017).

Para localização dos estudos foi consultada a Biblioteca Virtual em Saúde (BVS) que incluí as bases de dados: Scientific Electronic Library Online (SciELo), Literatura Latino-americana e do Caribe em Ciências da Saúde (LILACS) e Base de dados em Enfermagem (BDEnf). Foram utilizados os seguintes descritores isolados e/ou de forma combinada: Aleitamento Materno; Desmame Precoce e Pré-Natal.

Os critérios de inclusão foram estudos publicados nos últimos oito anos (2013 - 2021), pesquisas originais publicadas no Brasil, nos idiomas português, inglês e espanhol, estar circunscrito à temática abordada e apresentar texto completo. Assim, foram identificadas 623.934 publicações e excluídas 622.237 após aplicação dos filtros com os critérios de inclusão, posteriormente, excluiu-se 1532 por serem estudos duplicados ou por fugirem da temática após leitura do título. Foram, também, excluídos 421 estudos após leitura do resumo por não atenderem aos objetivos propostos, e por fim, excluiu-se seis após leitura do texto completo por não responderem a questão norteadora deste estudo. Logo, a revisão integrativa foi operacionalizada com oito estudos.

\section{Resultados}

O trabalho constitui-se de uma amostra variada composta de oito artigos, apresenta periódicos nacionais, nos quais dois estudos foram publicados em periódicos da área médica pediatra, um estudo na área de enfermagem, um na área de odontologia, um de epidemiologia, um de psicologia, e um em revista multidisciplinar. Observa-se diante da diversidade de publicações que o desmame precoce é uma questão multidisciplinar, cujo, profissionais de diversas áreas já se interessam em buscar os motivos que levam ao desmame para elaborar estratégias que mudem essa prática.

A Tabela 1, contém a síntese dos resultados encontrados e as características dos estudos quanto ao ano, periódico e metodologia. Dois estudos são de natureza qualitativa, e cinco quantitativa, fato que pode ser explicado pelos fatores constituírem questões objetivas.

Tabela 1 - Síntese dos resultados encontrados.

\begin{tabular}{|c|c|c|c|}
\hline Autor/Ano & $\begin{array}{c}\text { Periódico/Estado } \\
\text { de estudo }\end{array}$ & Método & Resultados \\
\hline Peres, JF; et al. (2021) & $\begin{array}{c}\text { Saúde em Debate } \\
\text { (Paraná) }\end{array}$ & Qualitativo & $\begin{array}{c}\text { Volta ao trabalho, mamilos invertidos, dor, fissuras, pouca produção de } \\
\text { leite materno, prescrição de suplementação por pediatras e crenças e } \\
\text { mitos atrelados a influência dos familiares. }\end{array}$ \\
\hline $\begin{array}{c}\text { Murari, CPC; et al. } \\
\text { (2021) }\end{array}$ & $\begin{array}{c}\text { Acta Paulista de } \\
\text { Enfermagem (São } \\
\text { Paulo) }\end{array}$ & Quantitativo & $\begin{array}{c}\text { Orientação e influência de terceiros e percepções das mães sobre as } \\
\text { necessidades do filho. }\end{array}$ \\
\hline $\begin{array}{c}\text { Lima, APC; et al. } \\
\text { (2018) }\end{array}$ & $\begin{array}{c}\text { J. Health Biol Sci } \\
\text { (BA) }\end{array}$ & $\begin{array}{c}\text { Revisão } \\
\text { integrativa da } \\
\text { Literatura }\end{array}$ & $\begin{array}{c}\text { Entre os fatores que dificultam a prática do aleitamento materno } \\
\text { encontrou-se: leite fraco/ insuficiente; pouco incentivo por parte dos } \\
\text { profissionais, falta de conhecimento da mãe, uso de chupeta, volta ao } \\
\text { trabalho, trauma mamilar, dor, aumento da idade da criança e baixa } \\
\text { escolaridade da mãe. }\end{array}$ \\
\hline $\begin{array}{c}\text { Alencar, AP; et al. } \\
\text { (2017) }\end{array}$ & $\begin{array}{c}\text { Rev Interdisciplinar } \\
\text { Saúde e Meio } \\
\text { Ambiente (Santa } \\
\text { Catarina) }\end{array}$ & Qualitativo & $\begin{array}{c}\text { Verificou-se que algumas mães possuem compreensão acerca da } \\
\text { importância do aleitamento materno, relataram receber informações } \\
\text { sobre a amamentação durante as consultas de pré-natal e apoio da } \\
\text { família. Entretanto, ficou evidenciado que seu conhecimento não foi o } \\
\text { suficiente para que elas mantivessem a amamentação. }\end{array}$ \\
\hline
\end{tabular}




\begin{tabular}{|c|c|c|c|}
\hline $\begin{array}{c}\text { Viana, RAA; et al. } \\
\text { (2014) }\end{array}$ & $\begin{array}{c}\text { Revista da ABENO } \\
\text { (Porto Alegre/ RS }\end{array}$ & Qualitativo & $\begin{array}{c}\text { A dúvida, insegurança, dor, fissura, cansaço e volta ao trabalho, } \\
\text { redução na produção de leite materno representam pontos de } \\
\text { dificuldade ao AMEX; }\end{array}$ \\
\hline $\begin{array}{c}\text { Oliveira, MGOA; et al. } \\
\text { (2013) }\end{array}$ & $\begin{array}{c}\text { Rev Bras de } \\
\text { Epidemiol (São } \\
\text { Paulo) }\end{array}$ & Quantitativo & $\begin{array}{c}\text { Mães com melhores condições socioeconômicas representadas pela } \\
\text { escolaridade, saneamento básico e posse de bens de consumo } \\
\text { apresentaram maior duração do aleitamento exclusivo/predominante em } \\
\text { ambos os municípios. Assistência pré-natal representada pelo maior } \\
\text { número de consultas influenciaram positivamente na duração do } \\
\text { aleitamento exclusivo/predominante. }\end{array}$ \\
\hline $\begin{array}{c}\text { Farias, SE; Wisniewski, } \\
\text { D. (2015) }\end{array}$ & $\begin{array}{c}\text { Revista Uningá } \\
\text { (Paraná) }\end{array}$ & Quantitativo & $\begin{array}{c}\text { A maioria das mães entrevistadas introduziu alimentos na dieta de seus } \\
\text { filhos antes dos seis meses em relação às dificuldades encontradas pelas } \\
\text { mulheres no período da amamentação, a maioria não encontrou } \\
\text { nenhuma dificuldade para amamentar, entretanto algumas relataram não } \\
\text { ter produzido leite suficiente para amamentar e outras relataram } \\
\text { problemas com as mamas, interrompendo o processo de amamentação. }\end{array}$ \\
\hline $\begin{array}{c}\text { Broilo, MC; et al. } \\
\text { (2013) }\end{array}$ & $\begin{array}{c}\text { Jornal de Pediatria } \\
\text { (RS) }\end{array}$ & Quantitativo & $\begin{array}{c}\text { Fatores sócioeconômicos e demográficos associados à adesão de } \\
\text { práticas alimentares saudáveis nos primeiros anos de vida } \\
\text { demonstraram a existência de associações entre a adesão e as } \\
\text { características maternas, tais como: idade, escolaridade, renda e } \\
\text { ocupação materna. }\end{array}$ \\
\hline
\end{tabular}

Fonte: RRCP et al. (2021).

\section{Discussão}

\subsection{Fatores que levam ao desmame precoce}

O desmame precoce, aquele preconizado pelo Ministério da Saúde que deve ser exclusivo até os seis meses e complementado até os dois anos, é multicausal. Ou seja, diversos são os fatores que influenciam ao desmame antes deste período preconizado, e esses fatores se associam favorecendo as "desculpas" utilizadas pelas mães para justificar este fato.

O estudo realizado por Bartolini et al. (2013) encontrou mães que substituíam a alimentação dos recém-nascidos (RN) por leite de vaca. Evidencia-se quão prejudicial é o leite de vaca para um RN, visto que é muito rico em gordura, proteínas e outros elementos que sobrecarregam o estômago da criança.

Observa-se que de sete estudos, quase metade dos estudos (três) apontam a introdução de outros leites, por baixa produção de leite como principal fator que leva ao desmame. Farias e Wisniewski (2015) encontraram que a maioria das mães introduziram outros alimentos na dieta dos filhos, devido as dificuldades encontradas, como a produção insuficiente de leite e “problemas" nas mamas. Este estudo encontrou também, que a ocupação da mãe contribui para que a amamentação exclusiva não se perpetue pelos seis meses.

A ocupação da mãe aparece como fator, juntamente com idade materna inferior a 18 anos, a baixa escolaridade e a baixa renda familiar. Três dos oito estudos apresentam esses fatores como causadores do desmame precoce. Broilo et al. (2013) afirma que as características socioeconômicas e demográficas da família, e principalmente, da mãe, está associada à adesão de práticas alimentares saudáveis nos primeiros anos de vida da criança.

O bico artificial é considerado um acalento para os bebês, principalmente, na ausência da mãe, por isso, é especialmente muito utilizado, e é um fator considerável para o desmame precoce, visto que, reduz o tempo de mamada do bebê, consequentemente a sucção e produção de leite materno. Outro fator é a dificuldade da mãe com a primeira amamentação, com o ensinar o RN a pegar na mama. Deve-se ressaltar quão importante é o profissional atuar na primeira mamada da criança, ensinando a mãe e a criança como se dá a amamentação. (Lima et al., 2018). Dessa forma, tanto a criança como a mãe podem sentir segurança e conforto nesse processo.

Ademais, aparecem associados ao desmame, o uso de álcool pela mãe, a realização de menos de seis consultas de prénatal, a posição da mamada, primipariedade e crença no leite fraco. Fatores esses que estão bastante ligados a falta de informação provocada pela falta de consultas pré-natais, e inexperiência com a primipariedade. Além disso, a falta de 
informação favorece a introdução de água, chás e outros líquidos (Lima et al., 2018).

Segundo o estudo de Alencar et al. (2017) as mães que deixaram de amamentar precocemente seus filhos foram por possuir a crença que o leite não supria a fome do bebê. O leite materno é o alimento mais saudável para RN, contém além dos nutrientes, as imunoglobulinas que protegem a criança, são os anticorpos passados de mãe para filho. Além disso, a amamentação favorece o vínculo entre mãe e filho. Explicações como essas devem ser repassadas nas consultas de pré-natal.

\subsection{Importância da amamentação e estratégias que favorecem o aleitamento materno exclusivo}

O leite materno é o principal alimento para o lactente, ele contém nutrientes importantes para crescimento, desenvolvimento, proteção e promoção da saúde das crianças (Bürger et al., 2021). Esse leite possui propriedade nutricionais adequadas para idade do bebê, visto que binômio mãe e filho se adéquam um ao outro, por exemplo, nos primeiros dias pósparto é um leite mais rico em proteínas e imunoglobulinas que garantem a proteção do bebê, posteriormente torna-se um leite mais rico em gordura, o qual favorece o ganho de peso da criança (Lima et al., 2018).

Dentre as estratégias para evitar o desmame prévio aos 06 meses, consiste na realização do máximo de consultas de pré-natal, e abordagem precoce dos profissionais para com a temática, informando as vantagens para binômio mãe-filho (Alencar et al., 2017). Para isso, torna-se fundamental que os profissionais estejam capacitados e cientes da importância de realizarem essas orientações e incentivo ao aleitamento materno a fim de garantir a promoção, proteção e apoio a amamentação (Lima et al., 2018).

Dessa forma, percebe-se a necessidade da ampliação do acesso ao pré-natal, além da realização de uma assistência em que sejam ofertadas à mãe todas as informações importantes sobre ela e seu filho. Nota-se também, uma falha na rede de proteção às mães que trabalham fora do domicílio, visto que a licença maternidade é apenas de três meses e elas precisam voltar ao trabalho e deixar seus filhos, dessa forma, é necessário orientar a esta mulher que ela tem direito a algumas saídas durante o horário de trabalho para amamentar seu filho (Alencar et al., 2017).

Vale ressaltar que abordar sobre aleitamento materno é sempre um tema que envolve não apenas a pesquisa científica, mas também, questões sociais, econômicas, psicológicas e culturais, oferecendo assim um campo de pesquisa multidisciplinar. Deve-se levar em consideração o grau de instrução da mãe, sua cultura e ensinamentos de família, para que os profissionais realizem educação em saúde de forma efetiva, com feedback das mães (Peres et al., 2021).

Outro ponto que deve ser orientado às mães, é quando a realização da ordenha e formas de armazenamento do leite para que possam ir trabalhar, bem como o direito a uma hora no tempo de serviço para amamentar seu filho (Farias e Wisniewski 2015).

\section{Conclusão}

Diante de tudo que foi exposto, verifica-se quão necessário é a assistência pré e pós-natal, o atendimento deve ser de forma holística, integral e multiprofissional. Deve-se identificar os fatores que levam ao desmame precoce e intervir através do acompanhamento e da educação em saúde. Dentre as causas do desmame precoce destacou-se os agentes socioeconômicos (nível de escolaridade, volta ao trabalho, gravidez na adolescência) e biológicos (mamilos invertidos, pega inadequada, fissuras e dor). Conclui-se também, a importância do desenvolvimento de estudos como este, que visam descobrir estes fatores que interferem na saúde da comunidade. Estimula-se também, o desenvolvimento de estudos que promovam novas estratégias para promover o AME e combater o desmame precoce com base nos agentes motivadores.

\section{Referências}

Abreu, F. C. P., Fabbro, M. R. C. \& Wernet, M. (2013). Fatores que intervêm na amamentação exclusiva: revisão integrativa. Rev Rene, 14(3), 610-619. 
Alencar, A. P., Nascimento, G., Lira, P., Fonseca, F., Fonseca, R. \& Alves, B. (2017). Principais causas do desmame precoce em uma estratégia de saúde da família. Saúde e meio ambiente: revista interdisciplinar, 6(2), 65-76.

Brasil. M. S. (2014). Saúde da criança: crescimento e desenvolvimento. Departamento de Atenção Básica.

Brasil. M. S. (2015). Saúde da criança: Aleitamento materno e alimentação complementar. Caderno de Atenção Básica no 23.

Brasil. M. S. (1993). Instituto Nacional de Alimentação e Nutrição-INAN. Secretaria de Programas Especiais-SPE. Programa Nacional de Incentivo ao Aleitamento Materno - PNIAM.

Broilo, M. C., Louzada, M. L. C., Drachler, M. L., Stenzel, L. M. \& Vitolo, M. R. (2013). Maternal perception and attitudes regarding healthcare professionals' guidelines on feeding practices in the child's first year of life. Jornal de Pediatria, 89(5), 485-491.

Bortolini, G. A., Vitolo, M. R. Gubert, M. B. \& Santos, L. M. P. (2013). Early cow's milk consumption among Brazilian children: results of a national survey. Jornal de pediatria, 89(6), 608-613.

Bürger, B., Schindler, K., Tripolt, T., Stüger, H. P., Wagner, K. H., Weber, A., \& Wolf-Spitzer, A. (2021). Breastfeeding Prevalence in Austria according to the WHO IYCF Indicators-The SUKIE-Study. Nutrients, 13(6), 2096.

Carvalho, J. L. D. S., Cirino, I. P., Lima, L. H. D. O., Sousa, A. F. D., Carvalho, M. F. D. \& Oliveira, E. A. R. (2016). Conhecimento das mães sobre aleitamento materno exclusivo e alimentação complementar. Saúde Redes, 383-392.

Farias, S. E., \& Wisniewski, D. (2015). Aleitamento materno x desmame precoce. Revista UNINGÁ Review, 22(1).

Feitosa, M. E. B., Silva, S. E. O. da, \& Silva, L. L. da. (2020). Breastfeeding: causes and consequences of early weaning. Research, Society and Development, 9(7), e856975071. https://doi.org/10.33448/rsd-v9i7.5071

Figueredo, S. F., Mattar, M. J. G. \& Abrao, A. C. F. D. V. (2013). Hospital Amigo da Criança: prevalência de aleitamento materno exclusivo aos seis meses e fatores intervenientes. Revista da Escola de Enfermagem da USP, 47(6), 1291-1297.

Hernandez, A. R. \& Köhler, C. V. F. (2011). Determinantes sociais do desmame: contribuições das diferentes abordagens metodológicas. Physis: Revista de Saúde Coletiva, 21, 937-954.

Lima, A. P. C., Nascimento, D. S. \& Martins, M. M. F. (2018). A prática do aleitamento materno e os fatores que levam ao desmame precoce: uma revisão integrativa. Journal of Health \& Biological Sciences, 6(2), 189-196.

Marques, E. S., Cotta, R. M. M. \& Priore, S.E. (2011). Mitos e crenças sobre o aleitamento materno. Ciência \& saúde coletiva, 16(5), $2461-2468$.

Mendes, K. D. S., Silveira, R. C. D. C. P. \& Galvão, C. M. (2008). Revisão integrativa: método de pesquisa para a incorporação de evidências na saúde e na enfermagem. Texto \& contexto-enfermagem, 17(4), 758-764.

Monteiro, J. C. S., Nakano, A. M. \& Gomes, F. A. (2011). O aleitamento materno enquanto uma prática construída: Reflexões acerca da evolução histórica da amamentação e desmame precoce no Brasil. Investigación y educación en enfermería, 29(2), 315-321.

Murari, C. P. C., Arciprete, A. P. R., Gomes-Sponholz, F. \& Monteiro, J. C. D. S. (2021). Introdução precoce da alimentação complementar infantil: comparando mães adolescentes e adultas. Acta Paulista de Enfermagem, 34.

Nascimento, V. C. D., Oliveira, M. I. C. D., Alves, V.H. \& Silva, K. S. D. (2013). Associação entre as orientações pré-natais em aleitamento materno e a satisfação com o apoio para amamentar. Revista Brasileira de Saúde Materno Infantil, 13(2), 147-159.

Oliveira, M. G. O. A. D., Lira, P. I. C. D., Filho, B. M. \& Lima, M. D. C. (2013). Fatores associados ao aleitamento materno em dois municípios com baixo índice de desenvolvimento humano no Nordeste do Brasil. Revista Brasileira de Epidemiologia, 16, 178-189.

Peres, J. F., Carvalho, A. R. D. S., Viera, C. S., Christoffel, M. M. \& Toso, B. R. G. D. O. (2021). Percepções dos profissionais de saúde acerca dos fatores biopsicossocioculturais relacionados com o aleitamento materno. Saúde em Debate, 45, 141-151.

Silva, W. F. D. \& Guedes, Z. C. F. (2013). Tempo de aleitamento materno exclusivo em recém-nascidos prematuros e a termo. Revista CEFAC, 15(1), 160171.

Sousa, M. S., Aquino, P. D. S., Aquino, C. B. D. Q., Penha, J. C. \& Pinheiro, A. K. B. (2015). Aleitamento materno e os determinantes do desmame precoce. Rev. enferm. UFPI, 19-25.

Sousa, L. M. M., Marques-Vieira, C. M. A., Severino, S. S. P. \& Antunes, A. V. (2017). A metodologia de revisão integrativa da literatura em enfermagem. Revista investigação em enfermagem. 17(21).

Viana, R. A., Ferreira, E. G., Barboza, M. C. C. \& Sampaio, L. M. A. (2014). Aleitamento materno: desmistificando esse ato de amor como uma abordagem na promoção da saúde. Revista da ABENO, 14(1), 38-46.

Vieira, G. O., Martins, C. D. C., Vieira, T. D. O., Oliveira, N. F. D. \& Silva, L. R. (2010). Factors predicting early discontinuation of exclusive breastfeeding in the first month of life. Jornal de pediatria, 86(5), 441-444. 